\title{
Stability and transformations of raspberry (Rubus idaeus L.) ellagitannins in aqueous solutions
}

\author{
Michał Sójka ${ }^{1} \mathbb{D} \cdot$ Michał Janowski $^{1} \cdot$ Katarzyna Grzelak-Błaszczyk $^{1}$
}

Received: 4 September 2018 / Accepted: 1 December 2018 / Published online: 18 December 2018

(c) The Author(s) 2018

\begin{abstract}
Ellagitannins are known to possess many beneficial and health-promoting properties, including antioxidant and antimicrobial effects, arising from the activity of both native compounds and the products of their degradation or metabolism. The wide range of beneficial properties is attributable to the great structural variety of these compounds, even though all of them belong to the same polyphenolic group, namely hydrolyzable tannins. Therefore, the potential of individual ellagitannins must be studied separately with the view to their application, as natural substances, in medicine or the food industry. The objective of the present work was to elucidate the effects of temperature and medium $\mathrm{pH}$ on the stability of the two main raspberry ellagitannins, i.e., lambertianin $\mathrm{C}$ and sanguiin $\mathrm{H}-6$, in aqueous solutions. Experiments were conducted within the temperature range of $20-80{ }^{\circ} \mathrm{C}$ and $\mathrm{pH} 2-8$ over $0-24 \mathrm{~h}$ of incubation. The content of the studied ellagitannins and the products of their decomposition was investigated using HPLC-DAD and LC-MS, respectively, with an Orbitrap detector. It has been found that the studied ellagitannins are stable in acidic conditions, but are rapidly degraded in neutral and mildly basic media at elevated temperature $\left(60-80^{\circ} \mathrm{C}\right)$. In mildly acidic conditions ( $\mathrm{pH}$ 6) ellagitannins hydrolyze to intermediate products, that is, sanguiin H-10 isomers, sanguiin H-2, and galloyl-HHDP-glucose isomers, with the main end products being ellagic and gallic acids. In addition to hydrolysis, ellagitannins may also undergo oxidation to compounds containing a dehydrohexahydroxydiphenoyl (DHHDP) group, also accompanied by the presence of brevifolin carboxylic acid, a product of DHHDP hydrolysis.
\end{abstract}

Keywords Ellagitannins · Stability · Hydrolysis · Lambertianin C · Sanguiin H-6

\section{Introduction}

Ellagitannins, which can be defined as polyphenols belonging to the group of hydrolyzable tannins, are naturally found in many plants and are known to possess a range of beneficial and health-promoting properties, including antioxidant, anti-inflammatory, and prebiotic activities [1-3]. These compounds are usually ingested by humans by the consumption of berries, such as strawberries, raspberries,

Michał Sójka

michal.sojka@p.lodz.pl

Michał Janowski

michal_janowski91@o2.pl

Katarzyna Grzelak-Błaszczyk

katarzyna.grzelak-blaszczyk@p.lodz.pl

1 Institute of Food Technology and Analysis, Lodz University of Technology, ul. Stefanowskiego 4/10, 90-924 Lodz, Poland and blackberries, both raw and processed. Ellagitannins are also found in walnuts, pomegranates, herbs, and, to a lesser extent, wines aged in oak barrels $[2,4,5]$. The daily consumption of ellagitannins as ellagic acid is estimated at $4-12 \mathrm{mg} /$ day, although data on this subject are scarce [2]. In chemical terms, ellagitannins are esters of hexahydroxydiphenic acid (HHDP) and saccharides (usually glucose). They occur in many forms since saccharide molecules may bind HHDP and gallic acid residues in different combinations, also creating oligomers [6]. It is generally acknowledged that ellagitannins are hydrolyzed by acids and bases, as a result of which HHDP residues split from the molecules and are transformed to ellagic acid. While this is true, this description is not very precise, as according to some reports in basic conditions ellagitannins may undergo numerous transformations resulting from hydrolysis, deprotonation, and oxidation, while in acidic media they remain relatively stable [7]. The best case in point is the natural fruit environment, which does not degrade 
ellagitannins, in contrast to high concentrations of mineral acids or trifluoroacetic acid, which are used for ellagitannin hydrolysis in analytical methods [8]. According to Appel [9], phenolic compounds undergo the most extensive transformations at $\mathrm{pH}>8$, where they assume ionic form (phenolate ions) or are converted to quinones as a result of oxidation, and are characterized by high reactivity conducive to degradation. Furthermore, another important factor crucial to ellagitannin stability is molecular structure. According to Tanaka et al. [10], ellagitannins are more readily soluble in water than gallotannins due to the HHDP moieties contained in the former. The hydroxyl groups in gallotannins form more hydrophobic and hydrogen bonds between molecules, which makes them more stable and less susceptible to degradation. Another major factor affecting polyphenolic stability is temperature. It is generally known that high temperature applied during technological processes or storage is conducive to polyphenolic degradation. Temperature significantly influences the stability of these compounds in fruits and fruit products characterized by low $\mathrm{pH}$, especially during storage. As reported by Qu et al. [11], ellagitannins such as punicalagin $\mathrm{A}$ and $\mathrm{B}$ obtained from pomegranate peel were degraded by more than $30 \%$ at $\mathrm{pH} 3.5$ and $37^{\circ} \mathrm{C}$ after 180 days. In the case of basic $\mathrm{pH}$ conditions, temperature is less important. In a study of Tuominen and Sundman [7], ellagitannins incubated at ambient temperature exhibited half-lives of less than $10 \mathrm{~min}$ at $\mathrm{pH} 10$. The above results indicate that ellagitannin degradation mostly depends on $\mathrm{pH}$ conditions. Due to the susceptibility of these compounds to hydrolysis at high $\mathrm{pH}$, they are readily degraded in the human gastrointestinal system to ellagic acid, which is subsequently metabolized to urolithins [4, 12]. Among the polyphenols present in raspberries, the largest group consists of ellagitannins, which account for $53-76 \%$ of the total, depending on the plant cultivar [13]. In turn, lambertianin $\mathrm{C}$ and sanguiin H-6 account for approx. $80 \%$ of total raspberry ellagitannins on average [14]. These compounds are trimers and dimers, respectively, of galloylbis-HHDP-glucose, a monomer in which the anomeric carbon $\mathrm{C} 1$ is bound to a gallic acid residue at position $\alpha$ or $\beta$ (forming potentillin and casuarictin, accordingly). The oligomerization of monomeric units occurs via oxidative $\mathrm{C}-\mathrm{O}$ coupling, in which the oxygen is donated by the hydroxyl group of the gallic acid residue of the first monomer, forming an ether bond with the HHDP residue of the other monomer [6].

Given the absence of literature data on the subject, the aim of this study was to determine the effects of parameters such as $\mathrm{pH}$, temperature, and incubation time on the stability of the main raspberry ellagitannins (lambertianin $\mathrm{C}$ and sanguiin H-6) in aqueous solutions. Furthermore, the products of the degradation of these ellagitannins were identified.

\section{Materials and methods}

\section{Plant material}

The study material was raspberry ellagitannin preparation (REP), which contained 44\% lambertianin $\mathrm{C}$ and $35 \%$ sanguiin H-6. The full method of REP production was detailed in our previous publication [15]. Briefly, raspberry pomace from juice manufacture was extracted with $60 \%$ acetone. Following acetone removal, the extract was injected onto a XAD 1600 N column (DOW, Midland, MI, USA) packed with Amberlite resin. The phenolic compounds retained in the column were eluted with ethanol at concentrations ranging from 10 to $60 \%$, obtaining fractions with different qualitative composition. Ellagitannins were obtained in the fraction eluted with $40 \%$ ethanol. This fraction was then subjected to alcohol removal by means of a rotary vacuum evaporator and freeze-dried to obtain REP, which was used in stability studies. The ellagitannin composition of REP is given in Table 1.

\section{Chemicals and standards}

Citric acid monohydrate and disodium phosphate for buffer preparation were obtained from POCH (Gliwice, Poland). For ellagitannin analysis, all solvents were HPLC or LC-MS grade. Acetonitrile and formic acid were purchased from Sigma-Aldrich Chemie (Steinheim, Germany), and orthophosphoric acid was from Avantor Performance Materials B.V. (Deventer, Holland). Lambertianin $\mathrm{C}$ and sanguiin $\mathrm{H}-6$ standards were prepared in our laboratory according to the procedure described in a previous work [13]. Ultrapure water for buffer preparation and

Table 1 Ellagitannin composition of raspberry ellagitannin preparation (REP)

\begin{tabular}{lll}
\hline Compound & Mean $(\mathrm{mg} / 100 \mathrm{~g})$ & $\mathrm{SD}$ \\
\hline Sanguiin H-10 isomer & 995 & 55 \\
Lambertianin C without ellagic moiety & 1397 & 98 \\
Sanguiin H-10 isomer & 698 & 42 \\
Sanguiin H-10 without ellagic moiety & $\mathrm{tr}$ & - \\
Lambertianin C without HHDP-HHDP- & $\mathrm{tr}$ & - \\
$\quad$ glucose & & \\
Lambertianin C & 43,650 & 1230 \\
Lambertianin C without ellagic moiety & $\operatorname{tr}$ & - \\
Galloyl-bis-HHDP-glucose & $\operatorname{tr}$ & - \\
Sanguiin H-6 & 35,040 & 1098 \\
Total ellagitannins & 81,780 & 3212 \\
\hline
\end{tabular}

Results are given as means of three replicates \pm standard deviation (SD)

tr trace amount, content not determined 
HPLC and LC-MS analysis was obtained from a Hydrolab HLP5 System (Straszyn, Poland).

\section{Stability studies of lambertianin C and sanguiin H-6}

Stability studies were conducted in $\mathrm{pH} 2,4,6,7$, and 8 buffers, which were obtained by mixing $0.1 \mathrm{M}$ citric acid and $0.2 \mathrm{M}$ disodium phosphate solutions at the following proportions ( $\mathrm{v} / \mathrm{v}$ at $20^{\circ} \mathrm{C}$ ): $3 / 0,2 / 1.5,1.1 / 2.4,0.9 / 2.6$, and $0.065 / 3$, respectively. The $\mathrm{pH}$ of each solution was tested using a $\mathrm{pH}$-meter. In the case of higher process temperatures, the proportions of the solutions were modified accordingly to ensure a desired $\mathrm{pH}$ value. Stability evaluation was performed for an ellagitannin solution with a concentration of approx. $200 \mathrm{mg} / \mathrm{L}$, which corresponds to that of raspberry juice [13]. For this purpose, $20 \mathrm{mg}$ of REP was weighed into a glass vessel, and $100 \mathrm{~mL}$ of a buffer solution with an appropriate $\mathrm{pH}$ was added. After the dissolution of the preparation, the sealed vessel was placed in an incubator. Incubation was conducted in four temperature variants, i.e., $20^{\circ} \mathrm{C}, 40^{\circ} \mathrm{C}, 60^{\circ} \mathrm{C}$, and $80^{\circ} \mathrm{C}$, for $24 \mathrm{~h}$. Samples for analysis were taken at the following incubation times: $0 \mathrm{~h}$ (immediately after REP dissolution), $1 \mathrm{~h}, 2 \mathrm{~h}, 4 \mathrm{~h}, 8 \mathrm{~h}$, and $24 \mathrm{~h}$. The samples were immediately diluted $1: 1(\mathrm{v} / \mathrm{v})$ with $0.1 \mathrm{M}$ citric acid solution to stabilize the ellagitannins, centrifuged at $10,000 \times g$, and transferred to chromatographic vials.

\section{Quantitation of ellagitannins}

The content of ellagitannins in incubated samples was determined according to procedure described previously by Sójka et al. [13]. Analysis were carried out using Smartline chromatograph (Knauer, Berlin, Germany) composed of a degasser (Manager 5000), two pumps (P1000), autosampler (3950), thermostat, and PDA detector (2800). Lambertianin $\mathrm{C}$ and sanguiin $\mathrm{H}-6$ were separated on Gemini C18 110 Å, $250 \mathrm{~mm} \times 4.6 \mathrm{~mm}$ i.d., $5 \mu \mathrm{m}$, column (Phenomenex, Torrance, CA, USA). The mobile phase consisted of solvent A $(0.05 \%(\mathrm{v} / \mathrm{v})$ phosphoric acid-water) and solvent B [83:17 (v/v) acetonitrile-water with $0.05 \%$ phosphoric acid]. The column temperature was set to $35{ }^{\circ} \mathrm{C}$, the flow rate was $1.25 \mathrm{~mL} / \mathrm{min}$, and the gradient program was as follows: $0-5 \mathrm{~min}, 5 \%(\mathrm{v} / \mathrm{v}) \mathrm{B} ; 5-10 \mathrm{~min}$, 5-15\% (v/v) B; 10-35 min, 15-40\% (v/v) B; 35-40 min, 40-73\% (v/v) B; 40-44 min, 73\% (v/v) B; 44-46 min, $73-5 \%(\mathrm{v} / \mathrm{v}) \mathrm{B}$; and $46-54 \mathrm{~min}, 5 \%(\mathrm{v} / \mathrm{v}) \mathrm{B}$. The injection volume was $20 \mu \mathrm{L}$. Data were collected using ClarityChrom v.3.0.5.505 software (Knauer, Berlin, Germany). Both compounds were detected at $250 \mathrm{~nm}$, and standard curves were used for quantitation.

\section{Identification of degradation products}

The degradation products of lambertianin $\mathrm{C}$ and sanguiin $\mathrm{H}-6$ were identified using LC-MS for the variant in which REP was incubated at $\mathrm{pH} 6$ and $80^{\circ} \mathrm{C}$. This variant was selected due to the fact that it was found to have the highest concentration of degradation products. Identification was carried out with the use of a Dionex Ultimate 3000 high performance liquid chromatograph (HPLC) coupled with a DAD and Q Exactive Orbitrap mass spectrometer (Thermo Fisher Scientific, Waltham, MA, USA). The mobile phase consisted of $1 \%(\mathrm{v} / \mathrm{v})$ formic acid in water (solvent $\mathrm{A}$ ) and 80:20 (v/v) acetonitrile-water (solvent B). The following gradient was used: 0-6.5 min, 5\% (v/v) B; 6.5-12.5 min, 5-15\% (v/v) B; 12.5-44 min, 15-45\% (v/v) B; 44-45 min, 45-75\% (v/v) B; 45-50 min, 75\% (v/v) B; $50-52 \mathrm{~min}, 75-5 \%(\mathrm{v} / \mathrm{v}) \mathrm{B}$; and 52-65 min, 5\% (v/v) B. The column used was a $250 \mathrm{~mm} \times 4.6 \mathrm{~mm}$ i.d., $5 \mu \mathrm{m}$, Luna C18(2) $100 \AA$ (Phenomenex, Torrance, CA). The column temperature was set to $35^{\circ} \mathrm{C}$, the flow rate was $1 \mathrm{~mL} / \mathrm{min}$, and the injection volume was $20 \mu \mathrm{L}$. Chromatographic data were collected using Xcalibur software (Thermo). The MS system coupled to the HPLC was an Orbitrap mass spectrometer equipped with an $\mathrm{H}$-ESI probe used in the negative mode. The source parameters were as follows: vaporizer temperature of $500{ }^{\circ} \mathrm{C}$, ion spray voltage of $4 \mathrm{kV}$, capillary temperature of $400{ }^{\circ} \mathrm{C}$; and sheath gas and auxiliary gas flow rates of 75 and 20 units, respectively. The detector was operated in either the full MS or full MS/dd-MS2 scan modes. In the full MS mode, the scan range of $m / z, 200-2000$ was used. To generate MS2 data, the full MS/ dd-MS2 scan mode was applied. The collision energy used to generate $\mathrm{MS}^{2}$ spectra was set to 20 . Tuning and optimization were performed using direct injection of REP diluted in an 80:20 (v/v) mixture of mobile phases $\mathrm{A}$ and $\mathrm{B}$ at a flow rate of $0.25 \mathrm{~mL} / \mathrm{min}$.

\section{Statistics}

Sanguiin H-6 and lambertianin C half-lives were analyzed using the $t$ test. To examine the course of ellagitannin hydrolysis, the peak areas of degradation products acquired in the selected ion monitoring (SIM) mode by the mass detector were analyzed using $k$-means clustering. Prior to analysis, the data obtained from the detector were standardized. Statistical analysis was conducted using Statistica v.10 software (StatSoft, Tulsa, OK).

\section{Results and Discussion}

\section{Kinetics of ellagitannin degradation}

Table 2 shows the obtained degradation rate constants $(k)$ and half-lives $\left(t_{1 / 2}\right)$ for sanguiin H-6 and lambertianin C. 
These values were calculated for conditions in which a decrease in ellagitannins was observed on the basis of fitting a first-order kinetic model to experimental data. Since in acidic media ( $\mathrm{pH} 2$ and 4$)$ at temperatures in the range of $20-60{ }^{\circ} \mathrm{C}$ the studied ellagitannins exhibited high stability, this kinetic model could not be used for $24 \mathrm{~h}$ incubation.

Analysis indicated that both parameters, that is, $\mathrm{pH}$ conditions and temperature, significantly affected the degradation rate of the examined ellagitannins, with the crucial factor being basic $\mathrm{pH}$ (Figs. 1, 2). At $\mathrm{pH} 8$, at all the temperature levels applied, both lambertianin $\mathrm{C}$ and sanguiin $\mathrm{H}-6$ underwent considerable degradation already during the first $2 \mathrm{~h}$ of incubation. At $20{ }^{\circ} \mathrm{C}$, the half-lives of the two compounds amounted to approx. $2.5 \mathrm{~h}$, while at $80{ }^{\circ} \mathrm{C}$ their values fell to less than $0.5 \mathrm{~h}$. A decrease in $\mathrm{pH}$ to 7 inhibited degradation, especially at $20^{\circ} \mathrm{C}$; at higher temperatures degradation processes decreased but did not cease completely. A further decline in $\mathrm{pH}$ led to greater stability of the studied ellagitannins. In mildly acidic conditions at ambient temperature, they were not degraded over $24 \mathrm{~h}$.

Nevertheless, degradation did occur at higher temperatures with the half-lives of both compounds at $60^{\circ} \mathrm{C}$ and $80^{\circ} \mathrm{C}$ decreasing to less than $2.5 \mathrm{~h}$. In the case of $\mathrm{pH}$ in the range of $2-4$, that is, in natural conditions for ellagitannins, the studied compounds exhibited high stability, especially at $20-60{ }^{\circ} \mathrm{C}$. At $80^{\circ} \mathrm{C}$, they were gradually degraded only after $4 \mathrm{~h}$ of incubation. It should also be noted that at $\mathrm{pH} 2$ and $80^{\circ} \mathrm{C}$ the half-lives of both compounds exceeded $60 \mathrm{~h}$. Similar results for raspberry ellagitannin hydrolysis were obtained by Daniel et al. [16], who observed a pronounced increase in ellagic acid concentration in neutral ( $\mathrm{pH} 7)$ and basic $(\mathrm{pH}$ 8) raspberry extract solutions. The same authors also reported high stability of raspberry ellagitannins in acidic conditions ( $\mathrm{pH} 2)$.

The ellagitannins investigated in this work revealed lower stability as compared to those examined by Tuominen and Sundman [7], who studied monomeric ellagitannins with lower molecular weight (digalloyl-4,6-HHDP-glucose, pedunculagin, and geraniin) consisting of one glucose molecule linked to HHDP, dehydrohexahydroxydiphenoyl (DHHDP), and/or gallic acid residues. Those compounds exhibited relatively high stability at $\mathrm{pH} 8$ at ambient temperature, with a degradation rate constant of less than 0.1 in those conditions. In our study, the reaction rate constants obtained in the same conditions ( $\mathrm{pH} 8$ and $20{ }^{\circ} \mathrm{C}$ ) for sanguiin H-6 and lambertianin $\mathrm{C}$ were 0.77 and 0.75 , respectively, and markedly increased at higher incubation temperatures. At $\mathrm{pH} 8$, for both compounds a temperature increase from $20^{\circ} \mathrm{C}$ to $40{ }^{\circ} \mathrm{C}$ or $60^{\circ} \mathrm{C}$ on average doubled the parameter $k$, while an increase from $20^{\circ} \mathrm{C}$ to $80^{\circ} \mathrm{C}$ led to an approx. sixfold increment in that parameter. A significant effect of temperature on the stability of monomeric ellagitannins was reported by Hemingway and Hillis [17]. In their study, at $\mathrm{pH} 8$ and $0{ }^{\circ} \mathrm{C}$ bis-HHDP-glucose did not exhibit any degradation after $3 \mathrm{~h}$ of incubation, while a temperature increase to $50{ }^{\circ} \mathrm{C}$ caused more than $80 \%$ degradation.

A comparison of the half-lives and degradation rate constants obtained in this study provides mixed results in terms of determining the ellagitannin with superior stability parameters. The $t$ test (Table 2) for the half-lives of sanguiin H-6 and lambertianin C did not reveal significant differences in four experimental variants, with lambertianin $\mathrm{C}$ exhibiting
Table 2 Rate constants $(k)$ and half-lives $\left(t_{1 / 2}\right)$ for the degradation of lambertianin $\mathrm{C}$ and sanguiin $\mathrm{H}-6$ at various temperatures and $\mathrm{pH}$ values

\begin{tabular}{|c|c|c|c|c|c|c|}
\hline \multirow[t]{2}{*}{ Conditions } & \multicolumn{3}{|c|}{ Lambertianin C } & \multicolumn{3}{|l|}{ Sanguiin H-6 } \\
\hline & $k\left(10^{-4} \mathrm{~s}^{-1}\right)^{\mathrm{a}}$ & $R^{2}$ & $t_{1 / 2}(\mathrm{~h})$ & $k\left(10^{-4} \mathrm{~s}^{-1}\right)^{\mathrm{a}}$ & $R^{2}$ & $t_{1 / 2}(\mathrm{~h})$ \\
\hline $20^{\circ} \mathrm{C}, \mathrm{pH} 7$ & $0.14 \pm 0.00$ & 0.995 & $13.32 \pm 0.07^{\mathrm{b}}$ & $0.13 \pm 0.00$ & 0.994 & $14.53 \pm 0.08^{b}$ \\
\hline $20^{\circ} \mathrm{C}, \mathrm{pH} 8$ & $0.75 \pm 0.00$ & 0.994 & $2.57 \pm 0.00^{\mathrm{b}}$ & $0.77 \pm 0.00$ & 0.999 & $2.51 \pm 0.00^{\mathrm{b}}$ \\
\hline $40^{\circ} \mathrm{C}, \mathrm{pH} 6$ & $0.06 \pm 0.00$ & 0.856 & $34.76 \pm 2.21^{\mathrm{b}}$ & $0.11 \pm 0.00$ & 0.993 & $18.08 \pm 0.36^{b}$ \\
\hline $40^{\circ} \mathrm{C}, \mathrm{pH} 7$ & $0.77 \pm 0.00$ & 0.978 & $2.50 \pm 0.02$ & $0.77 \pm 0.00$ & 0.995 & $2.51 \pm 0.00$ \\
\hline $40^{\circ} \mathrm{C}, \mathrm{pH} 8$ & $1.26 \pm 0.02$ & 0.974 & $1.53 \pm 0.02^{\mathrm{b}}$ & $1.85 \pm 0.01$ & 0.990 & $1.04 \pm 0.00^{\mathrm{b}}$ \\
\hline $60^{\circ} \mathrm{C}, \mathrm{pH} 6$ & $0.78 \pm 0.00$ & 0.958 & $2.46 \pm 0.01$ & $0.78 \pm 0.00$ & 0.996 & $2.47 \pm 0.01$ \\
\hline $60^{\circ} \mathrm{C}, \mathrm{pH} 7$ & $1.04 \pm 0.01$ & 0.996 & $1.84 \pm 0.01^{\mathrm{b}}$ & $1.51 \pm 0.01$ & 0.990 & $1.28 \pm 0.01^{\mathrm{b}}$ \\
\hline $60^{\circ} \mathrm{C}, \mathrm{pH} 8$ & $1.29 \pm 0.00$ & 0.987 & $1.49 \pm 0.00^{\mathrm{b}}$ & $1.48 \pm 0.00$ & 0.991 & $1.30 \pm 0.00^{\mathrm{b}}$ \\
\hline $80^{\circ} \mathrm{C}, \mathrm{pH} 2$ & $0.03 \pm 0.01$ & 0.920 & $70.16 \pm 19.15$ & $0.03 \pm 0.01$ & 0.949 & $65.24 \pm 16.64$ \\
\hline $80^{\circ} \mathrm{C}, \mathrm{pH} 4$ & $0.18 \pm 0.03$ & 0.974 & $10.83 \pm 1.91$ & $0.19 \pm 0.04$ & 0.976 & $10.37 \pm 1.94$ \\
\hline $80^{\circ} \mathrm{C}, \mathrm{pH} 6$ & $0.78 \pm 0.00$ & 0.994 & $2.48 \pm 0.01^{\mathrm{b}}$ & $1.00 \pm 0.01$ & 0.991 & $1.93 \pm 0.01^{\mathrm{b}}$ \\
\hline $80^{\circ} \mathrm{C}, \mathrm{pH} 7$ & $1.04 \pm 0.01$ & 0.999 & $1.86 \pm 0.02^{\mathrm{b}}$ & $1.19 \pm 0.00$ & 0.960 & $1.62 \pm 0.01^{\mathrm{b}}$ \\
\hline $80^{\circ} \mathrm{C}, \mathrm{pH} 8$ & $4.78 \pm 0.00$ & 0.913 & $0.40 \pm 0.00^{\mathrm{b}}$ & $4.44 \pm 0.01$ & 0.940 & $0.43 \pm 0.00^{\mathrm{b}}$ \\
\hline
\end{tabular}

$R^{2}$ of the curve determined from the plot $\ln \left(A / A_{0}\right)$ versus incubation time

Values of $k$ and $t_{1 / 2}$ are expressed as means of two replicates \pm standard deviation

${ }^{a}$ Half-life was calculated according to the formula $t_{1 / 2}=0.693 / \mathrm{k}$

${ }^{b}$ Half-lives of lambertianin C and sanguiin H-6 marked with two asterisks differ statistically 

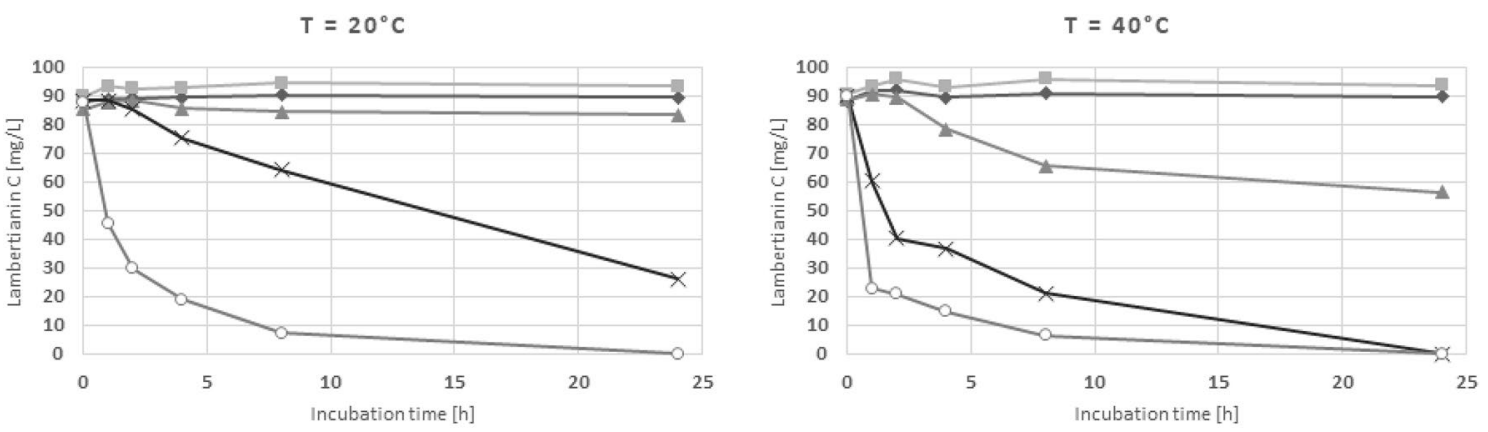

$\mathrm{T}=60^{\circ} \mathrm{C}$
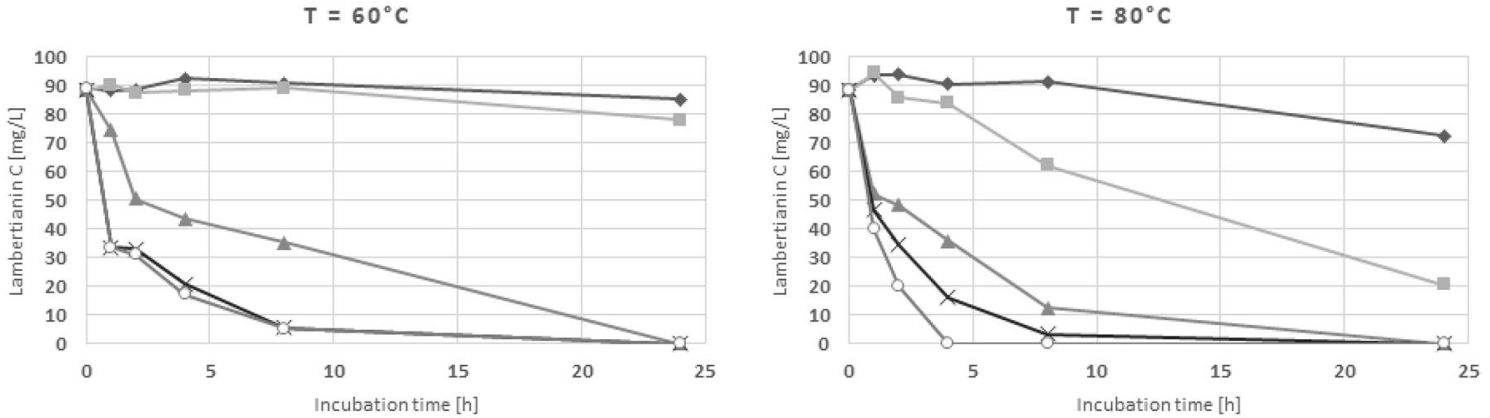

$\rightarrow \mathrm{pH}_{2} \rightarrow-\mathrm{pH} 4 \rightarrow \mathrm{pH} 6 \rightarrow \mathrm{pH} 7 \rightarrow \mathrm{pH} 8$

Fig. 1 Effects of $\mathrm{pH}$ and temperature on lambertianin $\mathrm{C}$ stability in aqueous buffer solutions. The presented data are means from two independent replicates
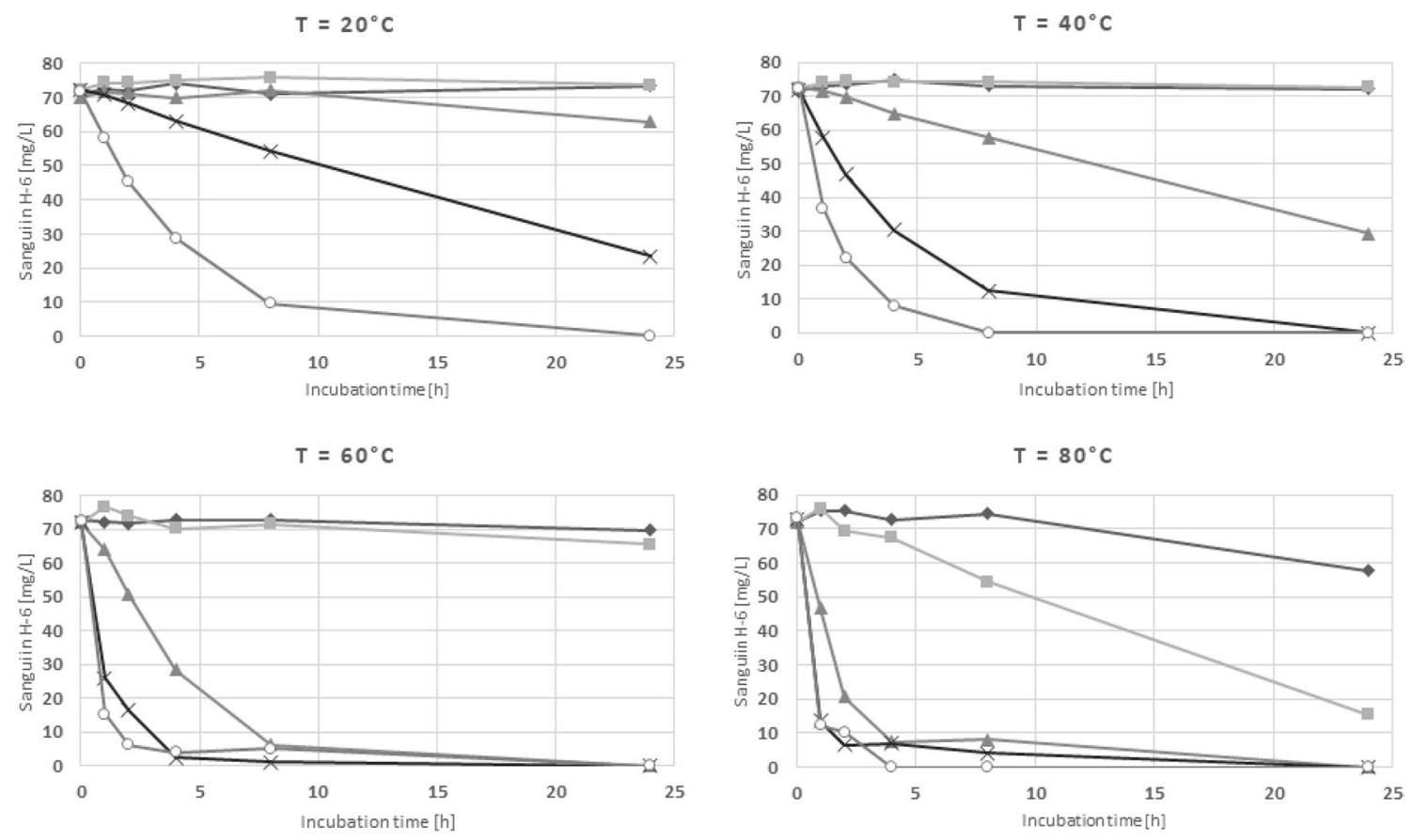

$\multimap \mathrm{pH}_{2}-\mathrm{pH} 4 \longrightarrow \mathrm{pH} 6 \multimap \mathrm{pH} 7 \multimap \mathrm{pH} 8$

Fig. 2 Effects of $\mathrm{pH}$ and temperature on sanguiin H-6 stability in aqueous buffer solutions. The presented data are means from two independent replicates 
higher stability in seven variants and sanguiin H-6 in two variants. It should also be noted that in many cases the halflives of these compounds were similar, with the differences possibly attributable to the calculations in the applied kinetic model.

Nevertheless, the presented data indicate that both of the studied dimeric and trimeric ellagitannins are less stable than monomeric ellagitannins and gallotannins. The study by Tuominen and Sundman [7] showed that monomeric ellagitannins, such as digalloyl-4,6-HHDP-glucose and pedunculagin, degraded in only approx. $10 \%$ after $6 \mathrm{~h}$ of incubation at $\mathrm{pH} 8$ at ambient temperature. Thus, it may be inferred that larger structures and higher molecular weights impair ellagitannin stability in basic conditions. According to Tanaka [10] and Tuominen and Sundman [7], the high stability of gallotannins is attributable to the greater number of hydrophobic and hydrogen bonds between their molecules in aqueous solutions, which in effect protects $\mathrm{OH}$ groups from deprotonation and further decomposition. In the case of ellagitannins, the presence of HHDP groups facilitates their solubility in water, thus increasing their susceptibility to hydrolysis.

\section{Degradation products of sanguiin H-6 and lambertianin C}

Qualitative analysis (LC-MS) was performed for samples incubated at $80{ }^{\circ} \mathrm{C}$ and $\mathrm{pH} 6$ for $0-24 \mathrm{~h}$. These conditions were selected because in quantitative analysis they had shown the greatest number of peaks arising from the degradation of native ellagitannins (Fig. 3). Table 3 presents the identification of 26 compounds found in REP before and after incubation. Native ellagitannins predominantly underwent hydrolysis to intermediate products, the most abundant of which were sanguiin $\mathrm{H}-10$ isomer, sanguiin $\mathrm{H}-2$, and galloyl-bis-HHDP-glucose isomers. The main end products of hydrolysis were ellagic and gallic acids (Fig. 4). In addition, we detected some products that may have been generated by oxidation, but their amount was much lower.

The incubated material, that is, REP, which is a highly concentrated ellagitannin preparation, contains nine ellagitannins, with the most abundant ones being lambertianin $\mathrm{C}$ (peak 19) and sanguiin H-6 (peak 23). The remaining seven constituents of REP, occurring in much lower amounts, are: two isomers of sanguiin H-10 (peaks 9 and 16), lambertianin $\mathrm{C}$ derivatives without an ellagic moiety (peaks 11 and 20), sanguiin H-10 without an ellagic moiety (peak 17), lambertianin C without HHDP-HHDP-glucose (peak 18), and galloyl-bis-HHDP-glucose (peak 22). Other compounds were detected only after incubation as they resulted from the hydrolysis or oxidation of the native ellagitannins.

Table 3 presents the responses of the mass detector to the content of the various compounds at different incubation times. These data show how their concentrations changed in the course of the process. To examine the hydrolysis of the studied ellagitannins, the data from the detector were analyzed using $k$-means cluster analysis (Fig. 5). The studied compounds have been grouped in 4 clusters. The first

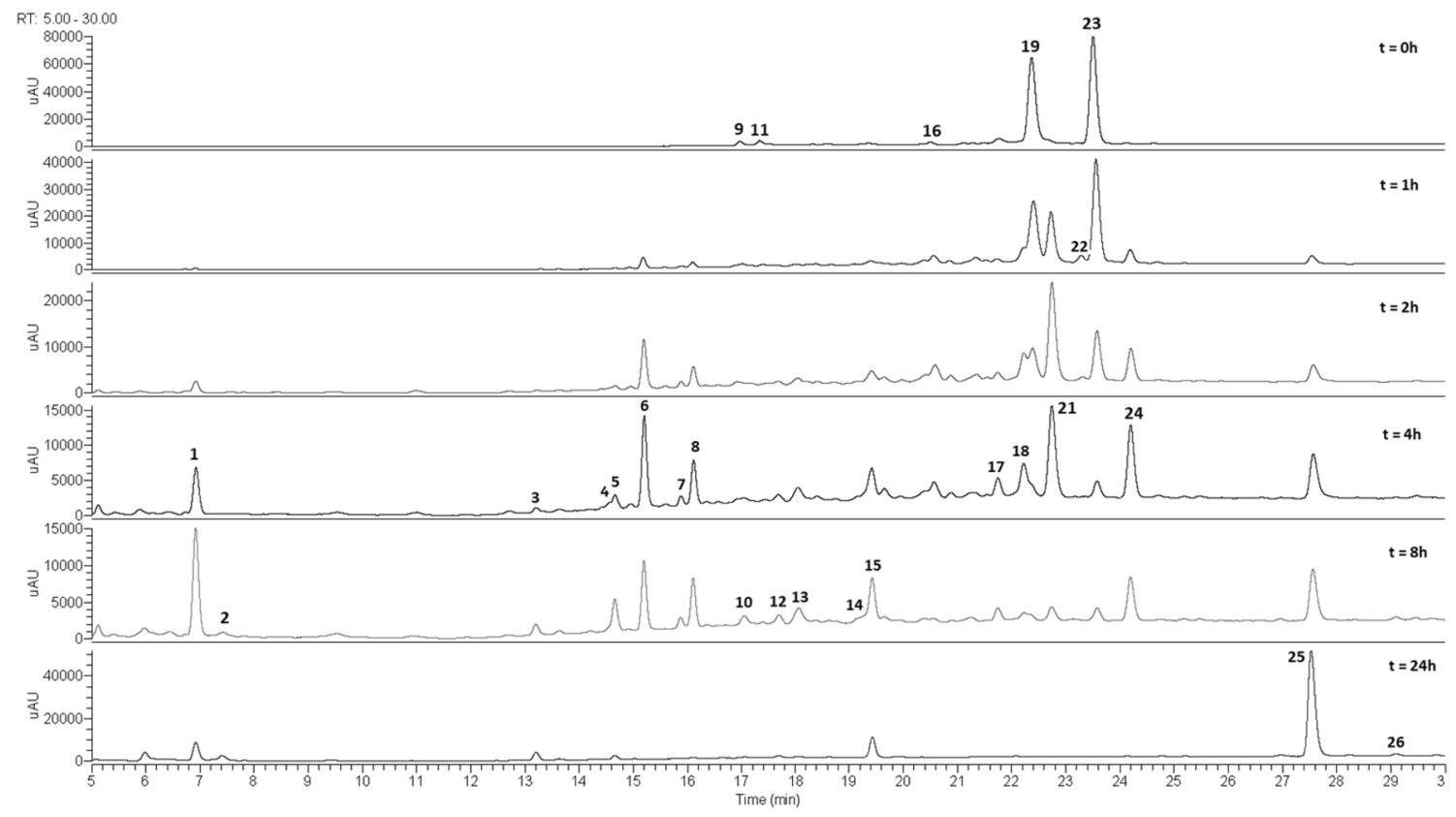

Fig. 3 Chromatogram $(250 \mathrm{~nm})$ of ellagitannins contained in REP and their degradation products for different incubation times at pH 6 and $80{ }^{\circ} \mathrm{C}$. Peak numbers correspond to those in Table 3 


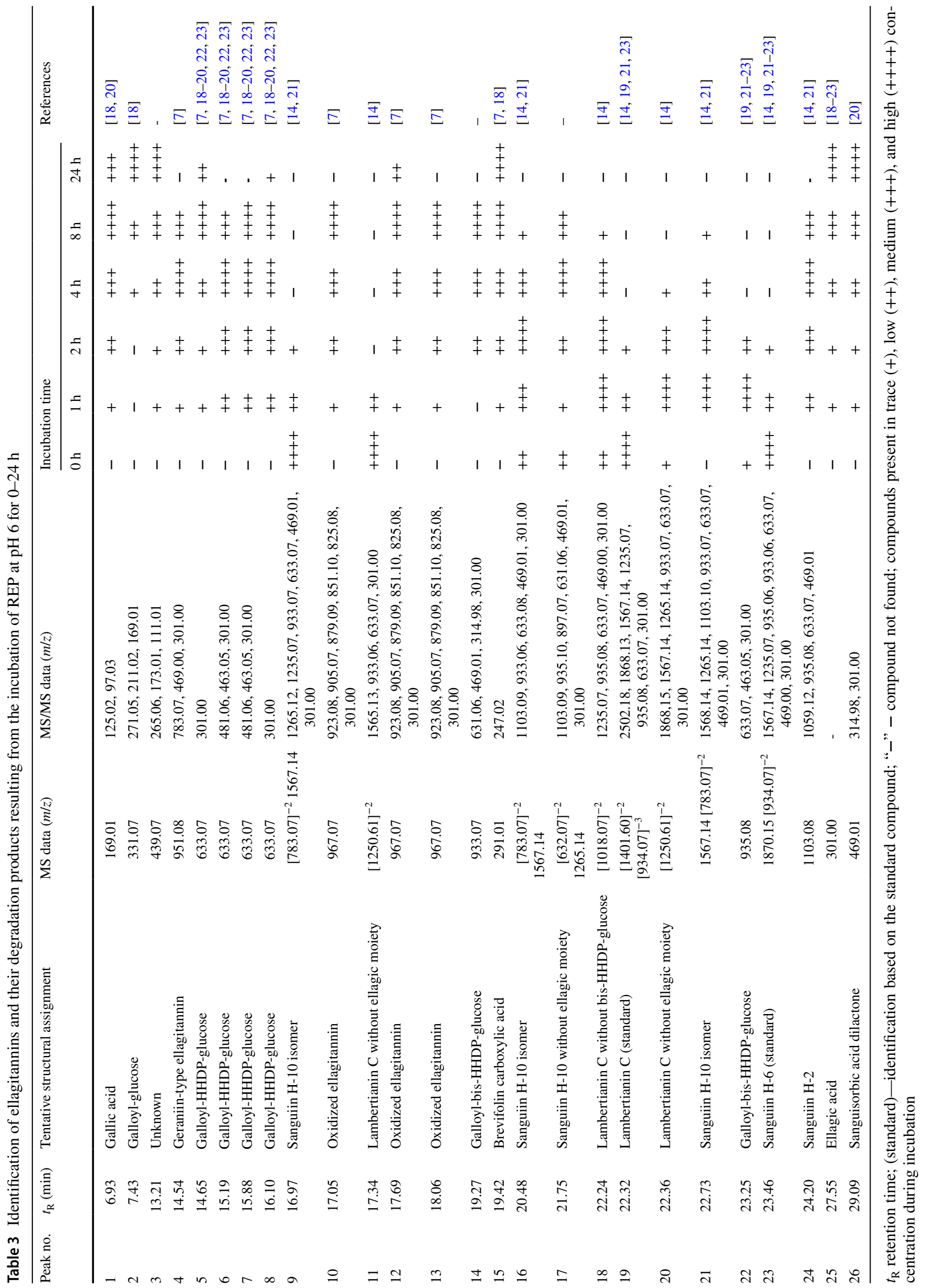


Fig. 4 The structure of lambertianin C, sanguiin $\mathrm{H}-6$, and their hydrolysis products

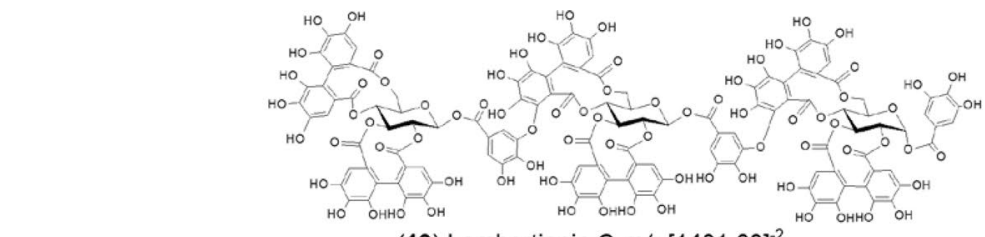

(19) Lambertianin C m/z [1401.60 $]^{-2}$

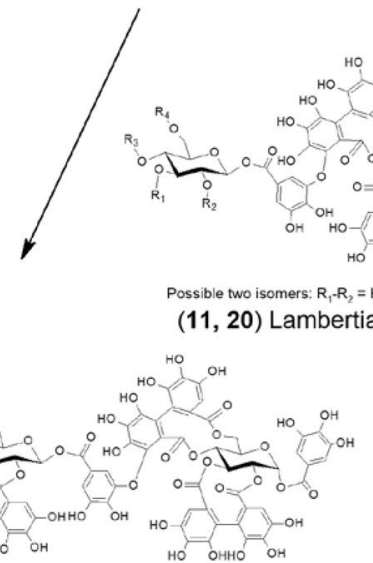

(18) Lambertianin C - HHDP-HHDP-glucose $\mathrm{m} / \mathrm{z}[1018.07]^{-2}$

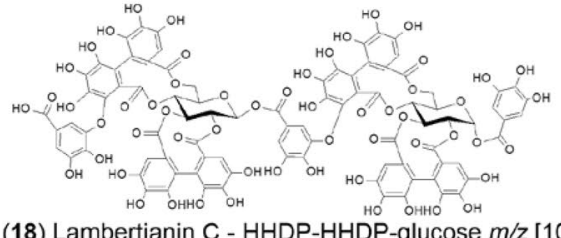

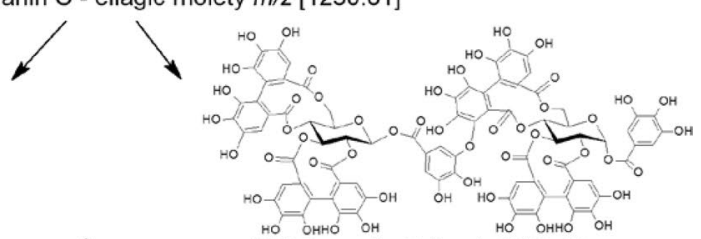

(23) Sanguiin $\mathrm{H}-6 \mathrm{~m} / \mathrm{z} 1870.15$

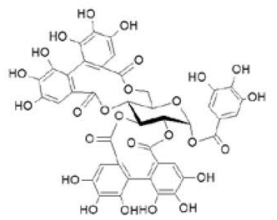

(22) Galloyl-bis-HHDP-glucose ( $\alpha$ or $\beta$ ) $\mathrm{m} / \mathrm{z} 935.08$

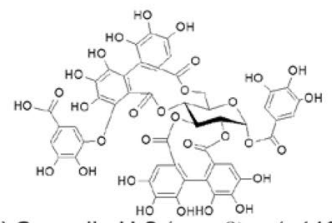

(24) Sanguiin $\mathrm{H}-2$ ( $\alpha$ or $\beta$ ) $\mathrm{m} / \mathrm{z} 1103.08$ next degradation products

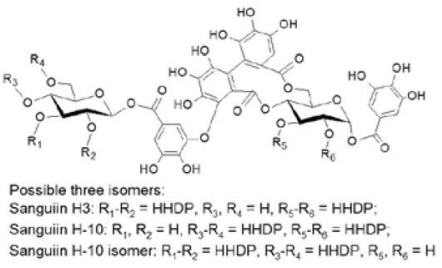

$(9,16,21)$ Sanguiin $\mathrm{H}-10$ isomers $m / z$ 1567.14

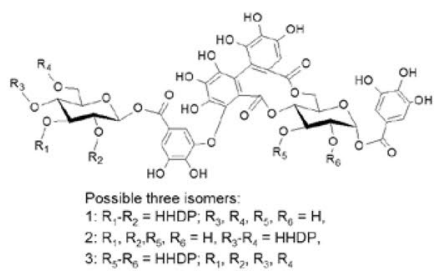

2: $R_{40}, R_{2}, R_{5}, R_{6}=H_{1}, R_{3}-R_{4}=H H D$
3: $R_{5}-R_{6}=H H D P ; R_{1}, R_{2}, R_{3}, R_{4}$

(17) Sanguiin $\mathrm{H}-10$ isomer - ellagic moiety $\mathrm{m} / \mathrm{z} 1265.14$ final degradation products

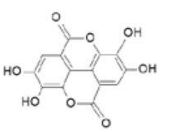

(25) Ellagic acid $\mathrm{m} / \mathrm{z} 301.00$
$(5,6,7,8)$ Galloyl-HHDP-glucose isomers $(\alpha$ and $\beta$ ) $m / z 633.07$ one consists of compounds which were degraded in the first stage of hydrolysis (peaks 9, 11, 19, and 23), that is, native ellagitannins. Their concentrations did not increase with incubation time.

The second and third clusters consist of intermediate products of early (1-2 h) and late hydrolysis (4-8 h). The second cluster contains compounds that arose due to the removal of an HHDP residue from sanguiin H-6 leading to sanguiin $\mathrm{H}-10$ isomers (peaks 16 and 21) as well as the removal of HHDP and HHDP-glucose residues (in that order) from lambertianin $\mathrm{C}$ leading to lambertianin $\mathrm{C}$ without an ellagic moiety (peak 20) and lambertianin $\mathrm{C}$ without bis-HHDP-glucose (peak 18, respectively). Another member of cluster 2 is galloyl-bis-HHDP-glucose (peak 22) resulting from the removal of this moiety from either lambertianin $\mathrm{C}$ or sanguiin H-6. The third cluster consists of compounds 
Fig. 5 Cluster analysis of ellagitannins contained in REP and their degradation products using $k$-means clustering

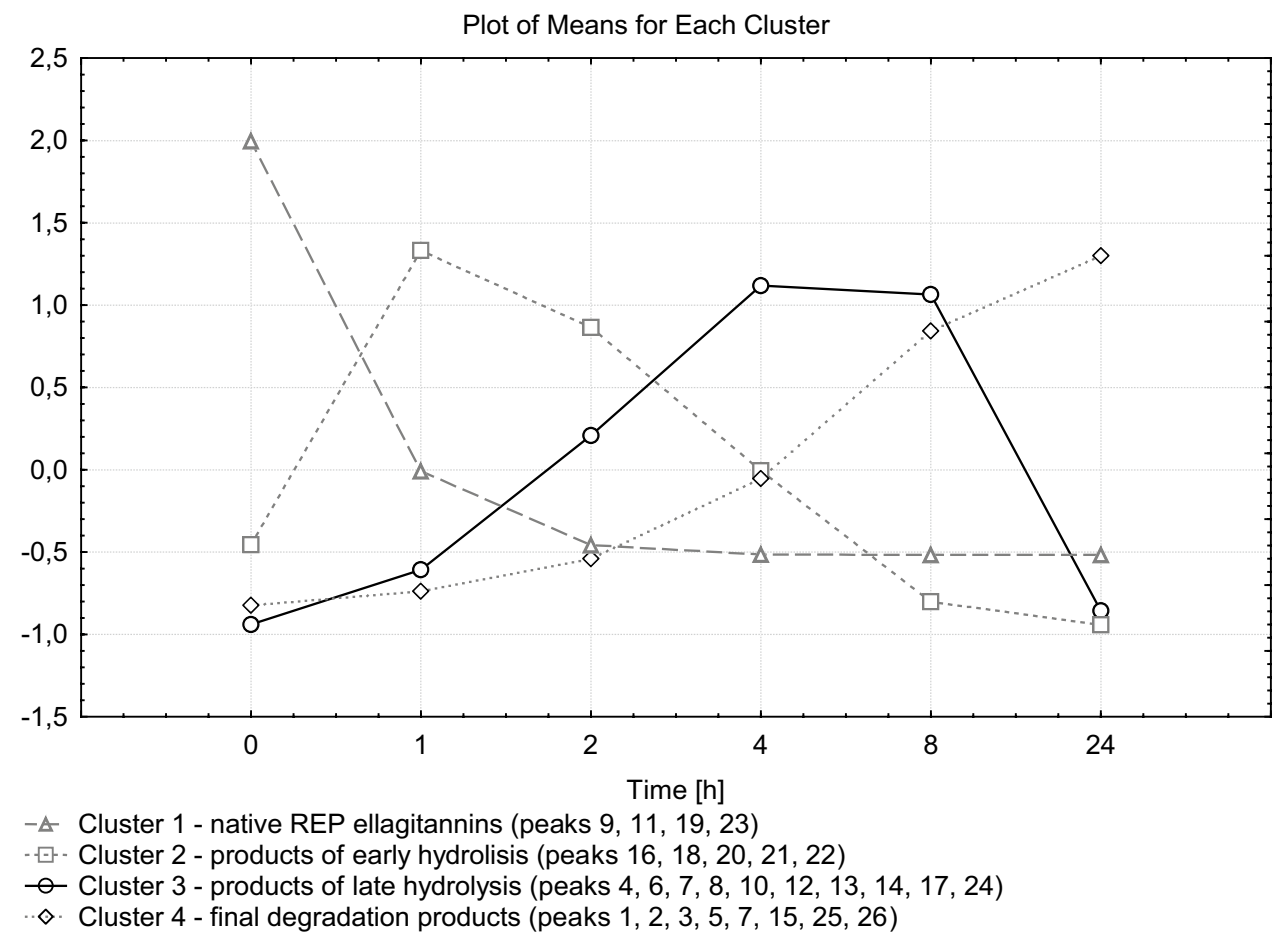

arising from further decomposition of those from cluster 2 , that is, sanguiin H-10 without an ellagic moiety (peak 17), sanguiin H-2 (peak 24) created by the removal of HHDP-glucose from sanguiin $\mathrm{H}-10$ isomers, and 3 isomers of galloyl-HHDP-glucose (peaks 6-8). The galloyl-HHDPglucose isomers generated at this stage of hydrolysis may occur in the form of anomers. In addition, cluster 3 reveals some compounds which may have arisen due to oxidation, such as galloyl-bis-HHDP-glucose (peak 14) producing pseudomolecular ion $\mathrm{m} / \mathrm{z} 933$ resulting from deprotonation and then oxidation to a semiquinone $[7,9]$. Other 3 oxidation products may be represented by peaks 10,12 , and 13. They gave molecular ion $\mathrm{m} / \mathrm{z}, 967$ and a characteristic sequence of fragment ions: $m / z$ 923, 879, and 851, resulting from the removal of $\mathrm{COOH}(\mathrm{m} / \mathrm{z} 44)$ and $\mathrm{C}=\mathrm{OCOOH}(\mathrm{m} / \mathrm{z}$ 72) groups. According to Tuominen and Sundman [7], these structures are attributable to further oxidation of quinones. Finally, cluster 3 features a compound (peak 4) defined as a geraniin-type ellagitannin, producing pseudomolecular ion $\mathrm{m} / \mathrm{z}$ 951. This compound is characterized by the presence of a DHHDP group resulting from the oxidation of a HHDP group. This is corroborated by the presence of brevifolin carboxylic acid (peak 15) in the hydrolysate, as this acid arises from hydrolysis of DHHDP [7, 24].

Finally, the fourth cluster consists of the end products of ellagitannin decomposition, including gallic acid (peak 1), galloyl-glucose (peak 2), galloyl-HHDP-glucose isomer (peak 3), brevifolin carboxylic acid (peak 15), ellagic acid (peak 25), and sanguisorbic acid dilactone (peak 26). All of them, except for brevifolin carboxylic acid, result from hydrolysis of native ellagitannins. The detected sanguisorbic acid dilactone indicates the presence of oligomeric ellagitannins-in this case lambertianin $\mathrm{C}$ and sanguiin H-6 [10]. This cluster also reveals a compound that could not be identified (peak 3), characterized by light absorption at $360 \mathrm{~nm}$ and pseudomolecular ion $\mathrm{m} / \mathrm{z}$ 439. Given its specific absorption properties, it may be a degradation product of an oxidized ellagitannin [7]. Three of the aforementioned compounds (gallic acid, ellagic acid, and sanguisorbic acid dilactone) were also identified by García-Villalba et al. [20], who conducted $24 \mathrm{~h}$ acidic hydrolysis of pomegranate extracts. Similarly as in the present study, those compounds were generated by the hydrolysis of sanguiin $\mathrm{H}-10$ and other native pomegranate ellagitannins.

The present study shows that the two main raspberry ellagitannins, that is, trimeric lambertianin $\mathrm{C}$ and dimeric sanguiin H-6 are stable in acidic conditions, but undergo rapid degradation in neutral and mildly basic conditions at elevated temperature $\left(60-80{ }^{\circ} \mathrm{C}\right)$. It has been shown that in mildly acidic conditions ( $\mathrm{pH} 6$ ) they hydrolyze, yielding intermediate products (mostly sanguiin $\mathrm{H}-10$ isomer, sanguiin H2, and galloyl-HHDP-glucose isomers), with the end products being ellagic and gallic acids. In addition to hydrolysis, the studied ellagitannins also undergo oxidation processes giving rise to molecules containing a DHHDP group, accompanied by its hydrolysis product, namely, brevifolin carboxylic acid.

Knowledge of these properties is crucial not only to raspberry ellagitannin applications in the food and pharmaceutical industries, but also in terms of the effects of neutral and 
basic conditions and high temperatures in biological studies of these compounds.

Acknowledgements This study was financially supported by the Statute Funds of the Institute of Food Technology and Analysis.

Author contributions Michał Sójka designed the research, supervised, performed the experiments and wrote the manuscript; Michał Janowski performed the experiments and analyzed the data, Katarzyna GrzelakBłaszczyk analyzed the data.

\section{Compliance with ethical standards}

Conflict of interest The authors declare that have no conflict of interest.

Compliance with ethics requirements This article does not contain any studies with human or animal subjects.

Open Access This article is distributed under the terms of the Creative Commons Attribution 4.0 International License (http://creativeco mmons.org/licenses/by/4.0/), which permits unrestricted use, distribution, and reproduction in any medium, provided you give appropriate credit to the original author(s) and the source, provide a link to the Creative Commons license, and indicate if changes were made.

\section{References}

1. Chung K-T, Wei C-I, Johnson MG (1998) Are tannins a doubleedged sword in biology and health? Trends Food Sci Technol 9:168-175

2. Landete JM (2011) Ellagitannins, ellagic acid and their derived metabolites: a review source, metabolism, function and health. Food Res Int 44:1150-1160

3. Bialonska D, Kasimsetty SG, Schrader KK, Ferreira D (2009) The effect of pomegranate (Punica granatum L.) byproducts and ellagitannins on growth on human gut bacteria. J Agric Food Chem 57:8344-8349

4. Lipińska L, Klewicka E, Sójka M (2014) Structure, occurrence and biological activity of ellagitannins: a general review. Acta Sci Pol Technol Aliment 13(3):289-299

5. Maier M, Oelbermann A-L, Renner M, Weidner E (2017) Screening of European medicinal herbs on their tannin content-new potential tanning agents for the leather industry. Ind Crops Prod 99:19-26

6. Okuda T, Yoshida T, Hatano T, Ito H (2009) In: Quideau S (ed) Ellagitannins renewed the concept of tannins. World Scientific Publishing, Singapore, 1:1-55

7. Tuominen A, Sundman T (2013) Stability and oxidation products of hydrolysable tannins in basic conditions detected by HPLC/ DAD-ESI/QTOF/MS. Phytochem Anal 24:424-435

8. Klimczak E, Król B (2010) Determination of different forms of ellagic acid in by-products from strawberry processing. Zywn Technol Jakosc 4(71):81-94

9. Appel HM (1993) Phenolics in ecological interactions: the importance of oxidation. J Chem Ecol 19(7):1521-1552
10. Tanaka T (2009) In: Quideau S (ed) Ellagitannins renewed the concept of tannins. World Scientific Publishing, Singapore, Ch. 4:119-151

11. Qu W, Li P, Hong J, Liu Z, Chen Y, Breksa AP III, Pan Z (2014) Thermal stability of liquid antioxidative extracts from pomegranate peel. J Sci Food Agric 94:1005-1012

12. Piwowarski JP, Granica S, Stefańska J, Kiss AK (2016) Differences in metabolism of ellagitannins by human gut microbiota ex vivo cultures. J Nat Prod 79:3022-3030

13. Sójka M, Macierzyński J, Zaweracz W, Buczek M (2016) Transfer and mass balance of ellagitannins, anthocyanins, flavan-3-ols, and flavonols during the processing of red raspberries (Rubus idaeus L.) to juice. J Agric Food Chem 64:5549-5563

14. Gasperotti M, Masuero D, Vrhovsek U, Guella G, Mattivi F (2010) Profiling and accurate quantification of Rubus ellagitannins and ellagic acid conjugates using direct UPLC-Q-TOF HDMS and HPLC-DAD analysis. J Agric Food Chem 58:4602-4616

15. Klewicka E, Sójka M, Klewicki R, Kołodziejczyk K, Lipińska L, Nowak A (2016) Ellagitannins from raspberry (Rubus idaeus L.) fruit as natural inhibitors of Geotrichum candidum. Molecules 21:908

16. Daniel EM, Ratnayake S, Kinstle T, Stronger GD (1991) The effects of $\mathrm{pH}$ and rat intestinal contents on the liberation of ellagic acid from purified and crude ellagitannins. J Nat Prod 54:4:946-952

17. Hemingway RW, Hillis WE (1971) Behavior of ellagitannins, gallic acid, and ellagic acid under alkaline conditions. Tappi J 54:933-936

18. Álvarez-Fernádez MA, Hornedo-Ortega R, Cerezo AB, Troncoso AM, García-Parrilla MC (2016) Determination of nonanthocyanin phenolic compounds using high-resolution mass spectrometry (UHPLC-Orbitrap-MS/MS) and impact of storage conditions in a beverage made from strawberry by fermentation. J Agric Food Chem 64:1367-1376

19. Dincheva I, Badjakov I, Kondakova V, Dobson P, McDougall G, Stewart D (2013) Identification of the phenolic components in Bulgarian raspberry cultivars by LC-ESI-MS ${ }^{\mathrm{n}}$. Int J Agric Sci Res 3(3): $127-138$

20. García-Villalba R, Espín JC, Aaby K, Alsalvar C, Heinonen M, Jacobs G, Voorspoels S, Koivumäki T, Kroon PA, Pelvan E, Saha S, Tomás-Barberán FA (2015) Validated method for the characterization and quantification of extractable and nonextractable ellagitannins after acid hydrolysis in pomegranate fruits, juices, and extracts. J Agric Food Chem 63:6555-6566

21. Kähkönen M, Kylli P, Ollilainen V, Salminen J-P, Heinonen M (2012) Antioxidant activity of isolated ellagitannins from red raspberries and cloudberries. J Agric Food Chem 60:1167-1174

22. Kajdžanowska M, Gjamovski V, Stefova M (2010) HPLCDAD-ESI-MS ${ }^{\mathrm{n}}$ identification of phenolic compounds in cultivated strawberries from Macedonia. Maced J Chem Chem Eng 29(2):181-194

23. Hager TJ, Howard LR, Liyanage R, Lay JO, Prior RL (2008) Ellagitannin composition of blackberry as determined by HPLC-ESIMS and MALDI-TOF-MS. J Agric Food Chem 56:661-669

24. Okuda T, Ito H (2011) Tannins of constant structure in medicinal and food plants-hydrolysable tannins and polyphenols related to tannins. Molecules 16:2191-2217 